\title{
The influence of anxiety on processing capacity for threat detection
}

\author{
Helen J. Richards • Julie A. Hadwin • Valerie Benson • \\ Michael J. Wenger • Nick Donnelly
}

Published online: 6 July 2011

(C) Psychonomic Society, Inc. 2011

\begin{abstract}
In the present study, we explored the proposition that an individual's capacity for threat detection is related to his or her trait anxiety. Using a redundant signals paradigm with concurrent measurements of reaction times and eye movements, participants indicated the presence or absence of an emotional target face (angry or happy) in displays containing no targets, one target, or two targets. We used estimates of the orderings on the hazard functions of the RT distributions as measures of processing capacity (Townsend \& Ashby, 1978; Wenger \& Gibson, Journal of Experimental Psychology. Human Perception and Performance, 30,708-719, 2004) to assess whether self-reported anxiety and the affective state of the face interacted with the level of perceptual load (i.e., the number of targets). Results indicated that anxiety was associated with fewer eye movements and increased processing capacity to detect multiple (vs. single) threatening faces. The data are consistent with anxiety influencing threat detection via a broadly tuned attentional mechanism (Eysenck, Derakshan, Santos, \& Calvo, Emotion, 7,336-353, 2007).
\end{abstract}

\section{H. J. Richards}

University of Southampton,

Southampton, England, UK

\section{J. A. Hadwin}

Developmental Brain-Behaviour Laboratory,

University of Southampton,

Southampton, England, UK

V. Benson $\cdot$ N. Donnelly

Centre for Visual Cognition, University of Southampton,

Southampton, England, UK

M. J. Wenger

University of Oklahoma,

Norman, OK, USA

\section{H. J. Richards $(\square)$}

Developmental Brain-Behaviour Laboratory,

School of Psychology, University of Southampton,

Southampton, UK

e-mail: hjr105@soton.ac.uk
Keywords Eye movements and visual attention - Reaction time methods Attention

Cognitive models suggest that anxiety is characterized by enhanced threat detection (e.g., Eysenck, 1992; Eysenck et al., 2007; Mogg \& Bradley, 1998). Some argue that, prior to threat detection, individuals with high levels of anxiety increase vigilance for threat by either maintaining a broad distribution of attention (Eysenck, 1992; Eysenck et al., 2007) or by excessively scanning the environment with rapid eye movements (Eysenck, 1992). Following threat detection, by these accounts, anxious individuals automatically narrow their attention to focus on threatening stimuli; this process results in reduced attention to other stimuli and is described as a selective attentional bias (Bar-Haim, Lamy, Pergamin, Bakermans-Kranenburg, \& van IJzendoorn, 2007; Mogg \& Bradley, 1998). There is considerable empirical support for selective attention to threat in anxiety (Bar-Haim et al., 2007); however, the relationship between anxiety, threat detection, and the distribution of attention remains unclear.

Empirical research on threat detection and the distribution of attention in anxiety has been carried out as two separate lines of enquiry. Visual search studies reveal enhanced detection of threat targets in anxious individuals (e.g., Byrne \& Eysenck, 1995; Matsumoto, 2010). Studies of detection and localization of peripheral stimuli reveal anxiety to enhance both (e.g., Keogh \& French, 1999; Shapiro \& Lim, 1989), although at a cost to attentional control (see Eysenck et al., 2007). To date, it is unclear whether the benefits of a broad focus of attention are particularly apparent in the detection of threatening (vs. nonthreatening) stimuli.

It is possible that the benefits of a broad focus of attention on threat detection can be demonstrated most effectively in specific experimental conditions. One way of exploring this issue is to consider the relationship between anxiety and threat detection when there is a possibility of 
threatening stimuli occurring in multiple locations. Previous research on threat detection in anxiety has typically considered performance in attentional tasks using singleton threat targets. However, a broadly tuned attention system may have developed to enable increased processing capacity to detect threat when appearing simultaneously and in more than a single location.

In the present study, we used a redundant signals paradigm (see Miller, 1982; Townsend \& Nozawa, 1995) to investigate how multiple threats are integrated across the visual field. In this paradigm, redundancy gains are revealed by mean RTs that are quicker in redundant(multiple) target trials than in single-target trials (e.g., Townsend \& Nozawa, 1995). In the context of threat detection, a redundancy gain could be driven by a system that operates across a broad focus, where it is possible to integrate threatening information from multiple locations within a processing architecture that allows coactivation. Under this account, evidence for the presence of threat accumulates more rapidly when there are multiple targets, leading to faster detection responses than the very fastest responses possible to single threats. However, other accounts (e.g., rapid scanning with eye movements or parallel but independent processing of targets) would also predict a redundancy gain in mean RTs.

To start to distinguish between these accounts, we used a set of orderings on the RT distributions to assess changes in processing capacity as a function of the number of targets (see Townsend \& Ashby, 1978; Townsend \& Nozawa, 1995; Wenger \& Gibson, 2004). Here, processing capacity refers to the resources or energy required to complete a task (in this case, to produce a detection response) in finite time (Wenger \& Gibson, 2004). Therefore, by considering the entire RT distribution, processing capacity measures move beyond measures of average speed to provide information about the work required to detect a target at each point in time (Wenger \& Gibson, 2004). If threatening information can be integrated from across the visual field, then less work should be required to detect threat at each point in time when there are multiple targets as compared with single targets. Additionally, we used the Miller inequality (i. e., the race model inequality; Miller, 1982) as a source of converging evidence for a coactive threat detection system. A violation of the Miller inequality indicates that RTs in the redundant-target condition are faster than those predicted by the fastest RTs in the single-target conditions; that is, there is coactivation between the two signals in a redundanttarget trial. Alternative accounts (e.g., rapid scanning or parallel processing of targets) would not predict these increases in processing capacity or violations of the Miller inequality.

We predicted that high levels of anxiety would be associated with enhanced threat detection, leading to the prediction of anxiety-related increases in processing capacity for multiple (vs. single) threats. In addition, we predicted that individuals with high levels of anxiety would maintain a broad allocation of attention to facilitate detection from all possible locations and that this would be associated with reduced, as opposed to increased (Eysenck, 1992), eye movements. We considered the specificity of enhanced threat detection to a particular form of anxiety by assessing the effects of trait, state, and social anxiety (social anxiety was conceptualized as two forms of social fear: fear of negative evaluation or fear when interacting with other people; see Mattick \& Clarke, 1998). Finally, we predicted that the distribution of attention would be under top-down control (Belopolsky, Zwaan, Theeuwes, \& Kramer, 2007) and, therefore, that high levels of self-reported attentional control would be linked to an ability to maintain a broad allocation of attention in order to maximize the chances of detecting targets (angry or happy) in multiple locations (i.e., increased processing capacity to detect multiple vs. single targets).

\section{Method}

\section{Participants}

Forty healthy adults (mean age $=22.00$ years, $S D=3.34$, range $=18-29$ years; 11 males) were compensated for their participation by either course credit or a small monetary incentive. All participants had normal or corrected-tonormal vision.

\section{Stimuli and apparatus}

Stimuli were color images of eight NimStim models (Tottenham et al., 2009) displaying angry, happy, and neutral expressions. Each face was set into an oval template and was presented against a black background. The faces were $165 \times 256$ pixels in size $\left(4.2^{\circ}\right.$ horizontally and $6.5^{\circ}$ vertically).

Trial displays contained two faces presented at 177 pixels $\left(4.5^{\circ}\right)$ to the right and left of central fixation. The identity of the face in the left position was independent of the identity of the face in the right position. Target-absent trials contained two neutral faces; single-target trials contained one emotional target face (angry or happy) and one neutral face; redundant-target trials contained two emotional target faces (either two angry faces or two happy faces).

The experiment was displayed on a 20-in. monitor $(1,280 \times 1,024$ resolution) at a viewing distance of $70 \mathrm{~cm}$. An Eyelink 1000 Desk Mount eyetracking system (SR 
Research Ltd.) recorded participants' vertical and horizontal eye movements. Pupil location was sampled monocularly at $1,000 \mathrm{~Hz}$.

\section{Questionnaires}

Participants completed the State-Trait Anxiety Inventory (STAI-S and STAI-T; Spielberger, 1983), the Fear of Negative Evaluation Scale (FNES; Watson \& Friend, 1969) and the Social Interaction Anxiety Scale (SIAS; Mattick \& Clarke, 1998). A total of $25-35 \%$ of the present sample reported symptoms on the STAI-T, FNES, and/or SIAS that were consistent with clinical levels of anxiety. Participants also completed the Attentional Control Scale (ACS; Derryberry \& Reed, 2002), which assesses the ability to focus and shift attention.

\section{Procedure}

Two practice blocks (one angry,one happy) containing 32 trials each were followed by four (two angry, two happy) randomized experimental blocks of 128 trials each. Block order was controlled by a Latin square, and each block contained 64 present (32 single, 32 redundant) and 64 absent trials. Targets appeared with equal probability in both locations.

Participants were instructed to indicate the presence or absence of angry or happy faces using a response box; response keys for presence and absence were counterbalanced across participants. A trial began with a central fixation cross for $1,000 \mathrm{~ms}$ or until the participant had fixated within one degree of the center of the screen for
$200 \mathrm{~ms}$. The trial display followed for 1,500 ms or until a keypress was made (whichever was earlier). The intertrial interval was 1,000 ms (see Fig. 1).

Participants completed the SIAS prior to the day of testing. The STAI-S was completed immediately before the eyetracking task, and the STAI-S, STAI-T, FNES, and ACS immediately following. Table 1 presents the descriptive statistics for the questionnaire measures. The internal reliability of these measures in the present sample was acceptable (Cronbach's $\alpha_{\mathrm{s}}>.85$ ).

\section{Results}

\section{RT analyses}

Exclusion criteria Only correct responses on present trials were analyzed following correction for fast guesses (Eriksen, 1988).

Redundancy gain The reciprocal of the RTs (Ratcliff, 1993) were analyzed using a 2 (number of targets: one vs. two) x 2 (expression: angry vs. happy) repeated measures ANOVA. The main effect of number of targets was significant, $F(1,39)=$ $102.95, p<.001, r=.22$ (see Table 2). RTs were shorter in the two versus the one target condition $(M=620.98, S D=74.12$ vs. $M=659.59, S D=79.37)$. The effect of expression and the interaction were not significant, $F_{s}<2$, ns. Stepwise regression analyses showed that trait anxiety, state anxiety, fear of negative evaluation, social interaction anxiety, and attentional control were not significant predictors of the reciprocal of the RTs in any condition.
Fig. 1 An example of the sequence of trial displays

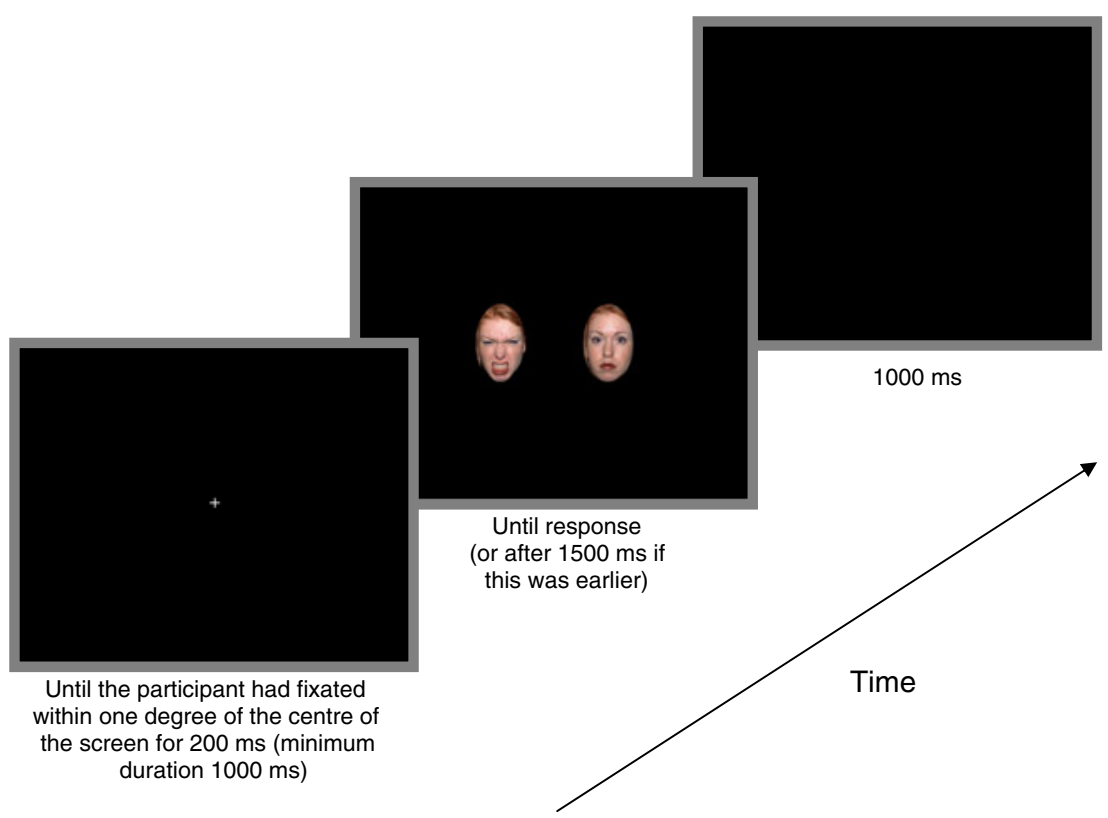


Table 1 Descriptive statistics for the questionnaire measures of individual differences

\begin{tabular}{lllll}
\hline & $M$ & $S D$ & Minimum (lower limit) & Maximum (upper limit) \\
\hline Trait anxiety (STAI-T) & 41.15 & 10.90 & $21(20)$ & $62(80)$ \\
Pre-test state anxiety (STAI-S) & 31.50 & 8.23 & $20(20)$ & $49(80)$ \\
Post-test state anxiety (STAI-S) & 32.98 & 9.40 & $20(20)$ & $55(80)$ \\
Social interaction anxiety (SIAS) & 23.48 & 12.07 & $7(0)$ & $57(76)$ \\
Fear of negative evaluation (FNES) & 18.18 & 7.64 & $3(0)$ & $29(30)$ \\
Attentional control (ACS) & 52.08 & 9.26 & $32(20)$ & $75(80)$ \\
\hline
\end{tabular}

Target redundancy and capacity Capacity was quantified at the level of the hazard function of the RT distribution; this provides a global measure of capacity at each point in time. Effects of the independent variables on the orderings of the hazard functions were assessed using the Cox proportional hazards model, stratifying the analyses by observer in order to deal with heterogeneity across observers (e.g., Therneau \& Grambsch, 2000). Schoenfeld residual plots revealed a departure from proportionality for the shortest $(<400 \mathrm{~ms})$ and longest ( $>725 \mathrm{~ms}$ ) RTs in the sample. Therefore, the analysis was repeated using only the RTs between 400 and $725 \mathrm{~ms}$, with number of targets, expression, and the interaction as predictors. Table 3 shows that the effect of the number of targets was significant, $\chi^{2}(1)=14.46, p<$ $.001, r=.60$, where an increase from one to two targets resulted in a $32 \%$ increase in processing capacity. There was a trend toward an effect of expression, $\chi^{2}(1)=3.25, p=.072$, $r=.29$, with a $15 \%$ increase in processing capacity for happy (vs. angry) faces; the interaction between expression and the number of targets was not significant $\chi^{2}(1)=1.15$, ns. All further analyses were carried out on angry and happy faces

Table 2 Mean and standard deviation of reaction times (in milliseconds), error rates (\%), and percentage of trials in which an eye movement occurred as a function of the number of targets and target expression

\begin{tabular}{lccccc}
\hline & \multicolumn{2}{l}{ Angry targets } & & \multicolumn{2}{l}{ Happy targets } \\
\cline { 2 - 3 } \cline { 5 - 6 } \cline { 5 - 6 } & $M$ & $S D$ & & $M$ & $S D$ \\
\hline Reaction times & & & & \\
Target absent & 670 & 95 & & 666 & 95 \\
Single target & 662 & 82 & & 657 & 86 \\
Redundant target & 626 & 75 & & 616 & 81 \\
Error rates & & & & \\
Target absent & 2.81 & 3.29 & 2.29 & 2.42 \\
Single target & 4.92 & 4.63 & 4.69 & 3.26 \\
Redundant target & 3.96 & 4.88 & 3.09 & 3.16 \\
Percentage of trials in which an eye movement occurred \\
Target absent & 66.84 & 34.64 & 66.44 & 34.00 \\
Single target & 71.59 & 32.67 & 69.62 & 33.63 \\
Redundant target & 65.73 & 36.28 & 65.91 & 36.78 \\
\hline
\end{tabular}

separately to account for the differences in processing capacity between expressions.

Capacity and anxiety The proportional hazards model was fit to the data of each observer individually, using number of targets as the predictor, and considering angry and happy faces separately. These individual estimates of the effect of the number of targets were regressed onto six possible predictors: trait anxiety, state anxiety (pre- and post-test measures of state anxiety were entered as separate variables), social-interaction anxiety, fear of negative evaluation, and attentional control. A stepwise model selection procedure was used to select the smallest set of predictors that would account for the largest proportion of the variance. For happy faces, none of the variables reached statistical significance. For angry faces, trait anxiety was a significant predictor of capacity changes $\left(\beta=0.195, R^{2}=\right.$ $0.57, p<.05$; see Fig. 2). This effect remained significant following Bonferroni correction for the two separate regression models. Thus, the increase in processing capacity associated with the addition of a redundant angry target was greater in individuals with higher levels of trait anxiety.

Converging evidence for the effect of trait anxiety was obtained using the Miller inequality. For every participant, empirical survivor functions $\widehat{S}(t)$ were estimated in $25-\mathrm{ms}$ intervals for each expression and each target present condition separately using the Kaplan-Meier method. The survivor functions were based on correct present responses from the uncensored data (i.e., RTs $<400 \mathrm{~ms}$ and $>725 \mathrm{~ms}$ were included). The empirical survivor functions give the probability that the manual response has not been made by time $t$ within the range of RTs observed for each participant. These empirical survivor functions were used in Equation 1 to calculate values of the Miller inequality in every 25-ms interval for angry and happy faces. Violations of the inequality (values $<0$ ) are indicative of coactive processing.

$\widehat{S}_{1 \& 2}(t)-\widehat{S}_{1}(t)-\widehat{S}_{2}(t)+1 \geq 0$,

where the subscript $1 \& 2$ indicates the redundant target condition, the subscript 1 indicates the single-target left 
Table 3 Results of fitting the proportional hazards model to the leftand right-censored RT data, stratifying across observers

\begin{tabular}{llllll}
\hline Predictor & $d f$ & $\widehat{\beta}$ & SE & $\chi^{2}$ & \% change \\
\hline Expression (E) & 1 & 0.14 & 0.07 & 3.25 & $15^{\dagger}$ \\
Load (L) & 1 & 0.28 & 0.07 & 14.46 & $32^{* * *}$ \\
Ex L & 1 & -0.05 & 0.05 & 1.15 & -4.9 \\
\hline
\end{tabular}

$* * * p<.001,^{\dagger} p=.072$

condition, and the subscript 2 indicates the single-target right condition.

Analyses using the Miller inequality were conducted in twenty separate time bins (T1-T20). For each participant, T1 was the first $25-\mathrm{ms}$ interval in which the value of the Miller inequality differed from 0 (i.e., where at least one of the three survivor functions differed from 1). The mean RT in T1 was $438.13 \mathrm{~ms}$ and $425.00 \mathrm{~ms}$ for angry and happy faces, respectively (thereafter, mean RTs increased by $25 \mathrm{~ms}$ per time bin). Stepwise regression analyses revealed that trait anxiety was the only significant predictor of Miller inequality values for angry faces in $\mathrm{T} 1\left(\beta=-.53, R^{2}=.28, p<.001\right)$, $\mathrm{T} 2\left(\beta=-.56, R^{2}=.31, \mathrm{p}<.001\right)$, and $\mathrm{T} 3\left(\beta=-.34, R^{2}=\right.$ $.12, p<.05$; see Fig. 3). There were violations of the Miller inequality in up to 21 participants in T1-T3 for angry faces, which is consistent with coactivation of multiple targets. Thus, at the earliest stages of the RT distribution, the ability to coactivate multiple threats was greater in individuals with high levels of trait anxiety. There were no statistically significant predictors in T4-T20 for angry faces. For the happy faces, although there were violations of the Miller inequality in up to 23 participants, there were no significant predictors in any time bin.

Errors The mean error rates were low at $2.55 \%$ ( $S D=$ $2.64), 4.81 \%(S D=3.28)$, and $3.53 \%(S D=3.36)$ for the

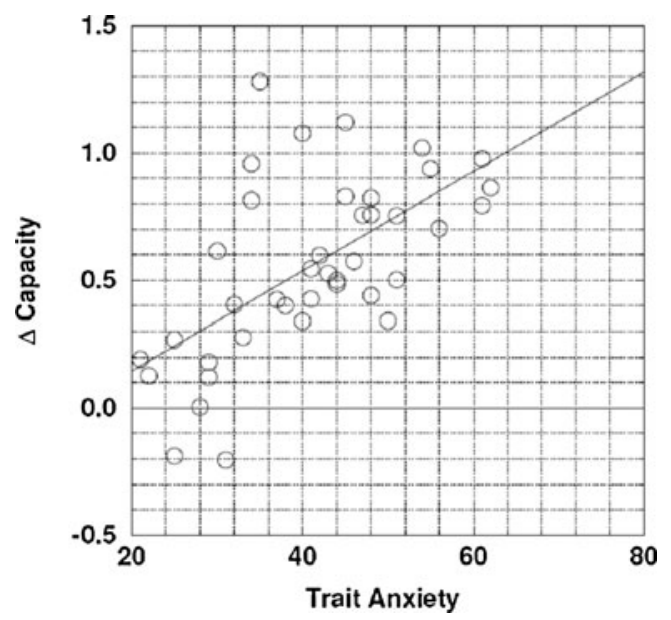

Fig. 2 Change in capacity (due to an increase in the number of targets) as a function of the trait anxiety score for angry faces target-absent, single-target and redundant-target conditions, respectively (see Table 2). Error rates were analyzed using the Wilcoxon signed rank test due to their skewed distribution. There was an effect of number of targets for each expression, where the error rate was significantly higher in the one-target condition than in the two-target condition, $z s>2, p s<.05, r \mathrm{~s}>.30$. There was no effect of expression on the error rate in the one or two target conditions $(z s<1, n s)$.

Eye movement analyses

Exclusion criteria Trials $(10.48 \%$ of the total) were removed from the eye-movement analysis if (a) the fixation location at the beginning of the trial was more than one degree away from the center of the screen; (b) an anticipatory eye movement occurred (defined as first saccades with latencies less than $80 \mathrm{~ms}$; Wenban-Smith \& Findlay, 1991); or (c) a blink occurred.

Eye movements and capacity Eye-movement trials were defined as those trials in which at least one eye movement was executed toward a target or distractor face with an amplitude greater than one degree. Table 2 indicates that eye movements were not necessary to acquire information about the presence or absence of a target (i.e., the eyes remained still on up to $35 \%$ of the trials). Analyses using the proportional hazards model (stratified across participants) revealed that the increase in processing capacity associated with an increase from one to two targets was numerically greater in trials in which the eyes remained still (a 33\% increase in processing capacity) as compared with eye-movement trials (a $23 \%$ increase in processing capacity).

Eye movements and trait anxiety Spearman correlations were conducted between scores on the STAI-T and the percentage of eye movement trials, considering each expression separately. For both angry and happy faces, there were significant negative correlations between trait anxiety and the percentage of trials in which an eye movement occurred for target-absent, single-target and redundant-target trials (all $r s<-.36$, all $p s<.05$ ); higher levels of trait anxiety were associated with fewer eye movements.

\section{Discussion}

A redundant signals study was used to explore the relationship between anxiety and processing capacity to detect threatening and nonthreatening faces. The results 

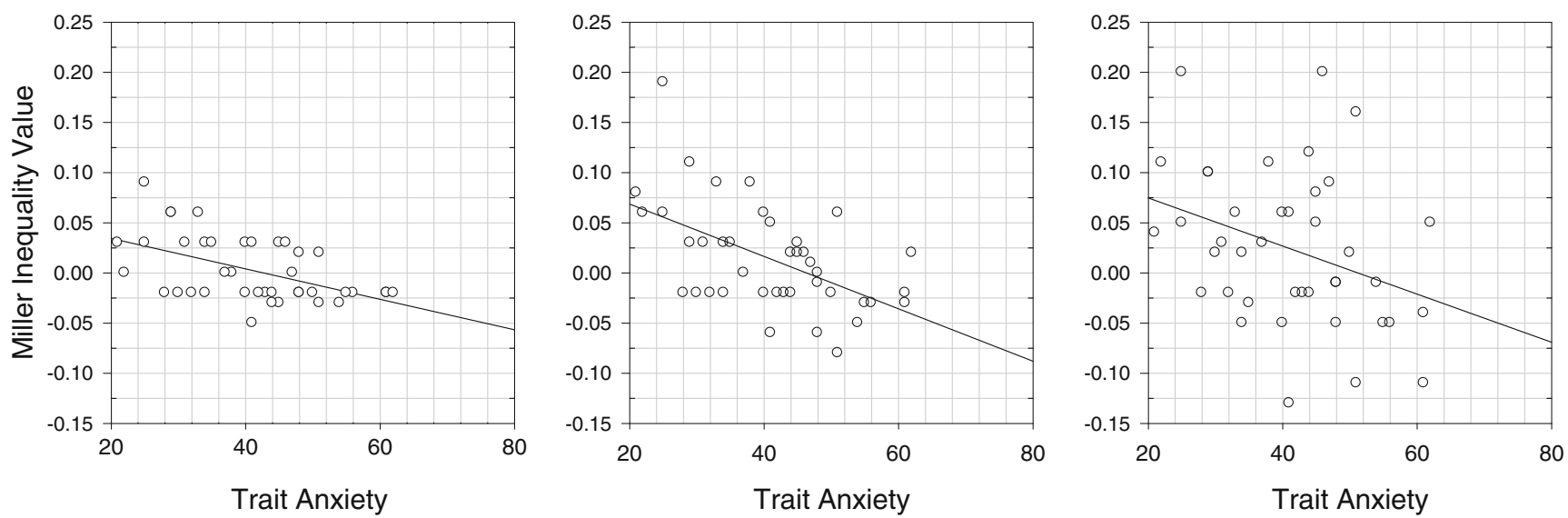

Fig. 3 Miller inequality values as a function of trait anxiety in T1 (left panel), T2 (middle panel), and T3 (right panel) for angry faces

revealed redundancy gains, in which mean RTs were faster in the presence of two (vs. one) targets. Furthermore, there was a $32 \%$ increase in processing capacity in the redundantas compared with the single-target condition. Thus, there was an improvement in detection performance (as indexed by processing capacity) with an increase in the number of targets, which is consistent with super-capacity processing (Townsend \& Nozawa, 1995).

Trait anxiety was associated with increased processing capacity to detect multiple (vs. single) angry faces in the context of reduced eye movements. This finding is consistent with the notion that there is a broad focus of attention in anxiety (e.g., Eysenck, 1992). The benefit of this broad focus of attention was to allow information from multiple threats to be pooled via coactivation from across the visual field so that responses to the presence of multiple targets were faster than to any one individual target (e.g., Miller, 1982; Townsend \& Nozawa, 1995). Importantly, high trait anxious individuals also executed fewer eye movements (but did not demonstrate increased processing capacity) when asked to detect happy faces. This finding raises the possibility that, even in the absence of threat, anxious individuals adopt a broad focus of attention in order to facilitate the detection of threatening stimuli in multiple locations and to minimize the potential danger associated with focusing attention on one object or location (Eysenck et al., 2007). These findings were specific to trait anxiety; increased processing capacity to detect angry faces was not observed in individuals with high levels of social or state anxiety.

The present findings inform theoretical models of anxiety by highlighting that neither selective attention (e. g., Mogg \& Bradley, 1998) nor lowered attentional control (Eysenck et al., 2007) provide a comprehensive account of the relationship between anxiety and attention. They indicate that there are beneficial effects of a broad focus of attention in anxiety, which are particularly apparent when coactive processing is required to integrate threatening information from multiple locations. Therefore, future work should consider the factors that enhance the efficiency of the coactive threat-detection system in individuals with high levels of anxiety.

A limitation in the present study design was that the presence of a target or a nontarget in one location provided probabilistic information about the presence or absence of a target in the other location. For example, if the left position contained a nontarget, then the right position was more likely to contain a nontarget than a target. In some situations, these interstimulus contingencies can lead to slowed detection in the single-target trials and facilitated detection in the redundant target trials (Mordkoff \& Yantis, 1991). Thus, although the results clearly indicate that anxiety is associated with enhanced threat detection in the presence of multiple threats, the present design leaves open the possibility that anxiety leads to an increased ability to use threat-related interstimulus contingencies rather than coactive threat detection.

We argue that the co-occurrence of increased processing capacity to detect multiple threats and fewer eye movements is most readily explained within a coactive processing account. It is also consistent with the theoretical notion that anxiety is linked to enhanced (rather than slowed) threat detection and a broad focus of attention (Eysenck, 1992). However, we fully accept that further empirical work is needed to confirm that anxiety is associated with coactive threat detection in the absence of interstimulus contingencies. Furthermore, it is clear that this future work might benefit from using two different types of nontarget to eliminate interstimulus contingencies while retaining an equal frequency of single and redundant target trials (Mordkoff \& Miller, 1993), and that nontargets should be low in emotional valence and distinct from both anger and happiness in emotional face space (e.g., low-intensity emotions such as neutral, tired, concerned, confused, and 
bored faces; Shah \& Lewis, 2003). Although challenging, the present results suggest that this would be an important study to conduct.

In summary, the present findings suggest that high levels of trait anxiety are associated with increased processing capacity for threat detection. Future models should consider not just the amplification of threat signals or lowering of threat-detection thresholds from single-threat targets but also the coactivation of signals from simultaneously presented threats. Together, these properties start to outline the fundamental attributes of a model for the role of anxiety on threat detection.

Author Note This research was supported by funding from the Economic and Social Research Council, U.K. (award number PTA031-2006-00179), and from the Worldwide Universities Network Research Mobility Programme.

We thank Kyle Cave, Katherine Cornes, Hayward Godwin, Tammy Menneer, and Rebecca Von Der Heide for their advice on previous drafts of this manuscript.

Some of the data from this study were presented in conference proceedings of the Vision Sciences Society Meeting, 2010.

\section{References}

Bar-Haim, Y., Lamy, D., Pergamin, L., Bakermans-Kranenburg, M. J., \& van IJzendoorn, M. H. (2007). Threat-related attentional bias in anxious and nonanxious individuals: A meta-analytic study. Psychological Bulletin, 133, 1-24.

Belopolsky, A. V., Zwaan, L., Theeuwes, J., \& Kramer, A. F. (2007). The size of an attentional window modulates attentional capture by color singletons. Psychonomic Bulletin and Review, 14, 934-938.

Byrne, A., \& Eysenck, M. W. (1995). Trait anxiety, anxious mood and threat detection. Cognition and Emotion, 9, 549-562.

Derryberry, D., \& Reed, M. A. (2002). Anxiety-related attentional biases and their regulation by attentional control. Journal of Abnormal Psychology, 111, 225-236.

Eriksen, C. W. (1988). A source of error in attempts to distinguish coactivation from separate activation in the perception of redundant targets. Perception \& Psychophysics, 44, 191-193.

Eysenck, M. W. (1992). Anxiety: The cognitive perspective. Hove, England: Erlbaum.

Eysenck, M. W., Derakshan, N., Santos, R., \& Calvo, M. G. (2007). Anxiety and cognitive performance: Attentional control theory. Emotion, 7, 336-353.
Keogh, E., \& French, C. C. (1999). The effect of trait anxiety and mood manipulation on the breadth of attention. European Journal of Personality, 13, 209-223.

Matsumoto, E. (2010). Bias in attending to emotional facial expressions: Anxiety and visual search efficiency. Applied Cognitive Psychology, 24, 414-424.

Mattick, R. P., \& Clarke, J. C. (1998). Development and validation of measures of social phobia scrutiny fear and social interaction anxiety. Behaviour Research and Therapy, 36, 455-470.

Miller, J. (1982). Divided attention: Evidence for coactivation with redundant signals. Cognitive Psychology, 14, 247-279.

Mogg, K., \& Bradley, B. P. (1998). A cognitive-motivational analysis of anxiety. Behaviour Research and Therapy, 36, 809-848.

Mordkoff, J. T., \& Miller, J. (1993). Redundancy gains and coactivation with two different targets: The problem of target preferences and the effects of display frequency. Perception \& Psychophysics, 53, 527-535.

Mordkoff, J. T., \& Yantis, S. (1991). An interactive race model of divided attention. Journal of Experimental Psychology. Human Perception and Performance, 17, 520-538.

Ratcliff, R. (1993). Methods for dealing with reaction time outliers. Psychological Bulletin, 114, 510-532.

Shah, R., \& Lewis, M. (2003). Locating the neutral expression in the facial-emotion space. Visual Cognition, 10, 549-566.

Shapiro, K. L., \& Lim, A. (1989). The impact of anxiety on visual attention to central and peripheral events. Behaviour Research and Therapy, 27, 345-351.

Spielberger, C. D. (1983). Manual for the State-trait anxiety inventory (Form Y). Palo Alto, CA: Consulting Psychologists Press.

Therneau, T. M., \& Grambsch, P. M. (2000). Modeling survival data: Extending the Cox model. New York, NY: Springer-Verlag.

Tottenham, N., Tanaka, J., Leon, A. C., McCarry, T., Nurse, M., Hare, T. A. et al. (2009). The NimStim set of facial expressions: Judgements from untrained research participants. Psychiatry Research, 168, 242-249.

Townsend, J. T., \& Ashby, F. G. (1978). Methods of modeling capacity in simple processing systems. In J. Castellan \& F. Restle (Eds.), Cognitive theory (Vol. III, pp. 200-239). Hillsdale, NJ: Erlbaum.

Townsend, J. T., \& Nozawa, G. (1995). Spatio-temporal properties of elementary perception: An investigation of parallel, serial and coactive theories. Journal of Mathematical Psychology, 39, 321-359.

Watson, D., \& Friend, R. (1969). Measurement of social-evaluative anxiety. Journal of Consulting and Clinical Psychology, 33, 448457.

Wenban-Smith, M. G., \& Findlay, J. M. (1991). Express saccades: Is there a separate population in humans? Experimental Brain Research, 87, 218-222.

Wenger, M. J., \& Gibson, B. S. (2004). Using hazard functions to assess changes in processing capacity in an attentional cuing paradigm. Journal of Experimental Psychology. Human Perception and Performance, 30, 708-719. 